\title{
Generación colaborativa de conocimiento. Una experiencia de aprendizaje basada en equipos o Team Based Learning (TBL) en la práctica odontológica.
}

\author{
Úrsula Muñoz $^{\mathrm{a}}$, Esther Escudero ${ }^{\mathrm{b}}$, Rima Barhoum ${ }^{\mathrm{c}}$, Maria de la Cruz Sádaba ${ }^{\mathrm{d}}$, Isabel \\ Sánchez-Vera ${ }^{\mathrm{e}}$.
}

aursula.munozmoron@ceu.es, ${ }^{\text {bestheresc@ceu.es, }}$,rbarho@ceu.es,

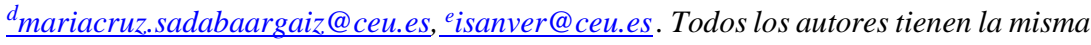
procedencia: Sección de Fisiología. Dpto. Ciencias Médicas Básicas. Facultad de Medicina. Universidad San Pablo CEU, Madrid.

\begin{abstract}
Lectureres teaching Physiology in the degree of Dentistry at the San Pablo CEU University have been involved in the past years in updating the teaching methodology in the field. The aim has been to favour the acquisition of several competencies by the students such us work autonomy along the progress of the subject, ability to look for and to find information from different sources, acquisition and use of several communication techniques and the implementation of collaborative work. The ultimate goal of these competencies is to broaden their abilities and deepen in knowledge acquisition so they become able to communicate and expand this knowledge in an academic environment. In this work we analyse one of these methodologies, an experience based in Team Based Learnign (TBL) in which additonallyy we have iused gamification learning techniques. The implementation of the new method has been carried out by using digital tools to facilitate information acquisition. The results obtained indicate that this new methodology increases the acquisition of knowledge, minimizes the time of work outside the classroom and improves their communication skills.
\end{abstract}

Keywords: team work, puzzle technique, expert groups ,test, polls, participation 
Generación colaborativa de conocimiento. Una experiencia de aprendizaje basada en equipos o Team Based Learning (TBL) en la práctica odontológica.

\begin{abstract}
Resumen
En la asignatura de Fisiología del Grado de Odontología los profesores están comprometidos de forma activa en actualizar la metodología docente a utilizar. En los últimos años, se ha fomentado que el alumno adquiriera distintas competencias como son: el aumento de su autonomía para gestionar su progreso en la asignatura, la búsqueda de información en fuentes diversas, el manejo de distintas modalidades de comunicación y el trabajo colaborativo. El objetivo de estas competencias está dirigido a que amplíen sus destrezas para profundizar en la adquisición de los conocimientos y que sean capaces de divulgarlos en el entorno académico. Con esta intención, en la asignatura de Fisiología, se han ofrecido a los alumnos distintas metodologías de aprendizaje. En la presente memoria se aborda el análisis de una de estas metodologías, la experiencia en el aprendizaje basada en equipos o Team Based Learning (TBL), dónde además se han ulizado técnicas de gammificación. La implementación de este método se ha llevado a cabo a través de herramientas digitales para facilitar la recogida sistémica de información. Los resultados obtenidos indican que esta nueva metodología aumenta la adquisición de conocimientos, minimiza el tiempo de trabajo fuera del aula y mejora sus capacidades comunicativas.
\end{abstract}

Palabras clave: Trabajo colaborativo y cooperativo, técnica puzzle, grupo de expertos, autoevaluación, encuestas, participación

\title{
Introducción
}

Para la adquisición de conocimiento por parte del alumnado es imprescindible el compromiso de realizar un trabajo autónomo de integración, comprensión, relación y almacenamiento de conceptos relacionados con el saber (Jensen et al., 2003). Además, es parte vertebral del aprendizaje que los conocimientos que se transmiten en clase sean asimilados, entendidos y relacionados de forma integral por los alumnos (Arceo et al., 2002). Por otro lado, es importante conseguir un abanico amplio de destrezas cognitivas y para ello es importante proporcionar métodos de aprendizaje activos de diferentes características (Zabalza, 2012), de tal manera que el proceso de aprendizaje no esté basado únicamente en el profesor como instructor, sino que en el aula se genere un binomio de "aprendizaje/facilitación del aprendizaje" (Barr and Tagg, 1995; García Valcárcel, 1993; Monereo y Pozo, 2003). Este último modelo está enfocado a favorecer la adquisición del saber a través de un aprendizaje autónomo de tal manera que el conocimiento se elabore de forma colaborativa entre iguales y que el profesor actúe como un mediador (Gargallo et al., 2015). Así el alumno pasa a estar directamente implicado en su proceso de aprendizaje investigando, cuestionando, haciendo aportaciones propias y siendo capaz de autoevaluarse y regular la evolución de su conocimiento (Machemer y Crawford, 2007).

En los últimos años, los profesores de la asignatura de Fisiología han pretendido implementar el método "aprendizaje/facilitación del aprendizaje" pretendiendo obtener distintas competencias por parte del alumnado como son: la búsqueda de información en diversas fuentes fiables, el manejo de distintas modalidades de comunicación, y el aumento de la autonomía para gestionar su progreso en la asignatura y el trabajo colaborativo. El objetivo

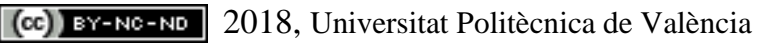

Congreso IN-RED (2018) 
ha estado dirigido a ampliar las destrezas de los alumnos para que profundicen en el conocimiento y a formarles como divulgadores de su pensamiento en el entorno académico. Con esta intención, se ha planteado que el alumno realice distintas actividades, que han sido:

- realización de autoevaluaciones en la plataforma BlackBoard,

- uso de un foro informático para la puesta en común de información aportada por los alumnos,

- cumplimentación de un cuestionario para valorar la utilizad docente de cada una de las actividades propuestas.

- manejo de motores de búsquedas informáticos y repositorios bibliográficos

- trabajos en equipo

- exposición de trabajos orales

Muchas de estas actividades ya se han puesto en marcha en nuestro Departamento con una gran aceptación por parte del alumnado y un aumento de su rendimiento académico. Un ejemplo de estas metodologías fue la realización de autoevaluaciones en la plataforma BlackBoard y el uso de un foro informático para la puesta en común de la información aportada por los alumnos (Escudero et al 2017; Sanchez-Vera et al 2017). Otras actividades, como la elaboración de seminarios por parte de los alumnos, están siendo modificadas con el objetivo de aumentar la adquisición de conocimientos, minimizar el tiempo de trabajo fuera del aula y mejorar sus capacidades comunicativas. Para poder llevar a cabo este objetivo hemos desarrollado una nueva metodología de aprendizaje siguiendo la experiencia en el aprendizaje basado en equipos o Team Based Learning (TBL) (Larry Michaelsen et al. 2004, Michaelsen y Sweet 2009, Estelles-Miguela et al 2017) que hemos modificado utilizando nuevas técnicas de aprendizaje basado en cooperación (Ricard, 2010).

Este tipo de metodologías nos ha permitido mejorar el aprendizaje colaborativo mediante la creación de equipos de alto rendimiento que cooperan entre ellos a la vez que compiten para demostrar el resultado de aprendizaje deseado. La implementación de este método para fomentar el estudio previo y el trabajo en equipo se lleva a cabo mediante los smartphones de los alumnos mediante programas como Socrative, Kahoot, o Quizizz. Este tipo de plataformas se utilizan en clase, permiten al alumno conocer las respuestas de forma inmediata y al profesor la recogida de resultados de forma automática. Además, consideramos muy interesante utilizar este tipo de metodologías de aprendizaje cooperativo, ya que, generan estructuras de interacción y de ayuda mutua en la que todos salen ganando. A veces para el alumno es más fácil cuando tienen dificultades acudir a un igual, el aprendizaje cooperativo ayuda a crear estas vías cercanas y alternativas al profesor. El aprendizaje mejora cuando enseñas y aprendes de otros.

\section{Objetivos}

El objetivo principal de este trabajo es proponer una nueva metodología de aprendizaje basada en equipos o Team Based Learning (TBL) junto con el uso de técnicas de aprendizaje cooperativo. 
para aumentar el porcentaje de aciertos en preguntas aplicadas sobre fisiología del sistema nervioso en las pruebas de evaluación continua realizadas a los alumnos de primer curso del Grado en Odontología.

Con la puesta en marcha de esta experiencia se buscaba dos objetivos concretos:

1. Aumentar la participación activa del alumno en la generación de nuevo conocimiento a partir de conocimientos aprendidos en el aula y fuera de ella.

2. Familiarizarse con preguntas aplicadas basadas en el conocimiento previo.

\section{Desarrollo de la innovación}

En esta memoria se presenta el análisis y las reflexiones obtenidas sobre la aplicación de una metodología basada en el Aprendizaje Basado en Equipos o Team Based Learning (TBL) en la preparación de un seminario sobre una aplicación clínica de una parte de los conocimientos desarrollados en la asignatura de Fisiología. Esta asignatura se imparte en el segundo semestre del $1^{\circ}$ curso del Grado en Odontología, es de carácter obligatorio y consta de 6 créditos ECTS. El estudio se ha realizado con los 84 alumnos matriculados en los dos grupos del curso académico 2017-18.

\section{Fase previa a la organización del seminario}

El tema propuesto para el seminario es el dolor orofacial. Este tema es uno de lo más importantes para los estudiantes de Odontología, ya que tienen que integrar los conocimientos aprendidos previamente acerca de la fisiología del sistema nervioso con una aplicación clínica como es el dolor orofacial y sus tratamientos. Por ello, en esta fase previa el profesor les explica en el aula todos los conceptos fundamentales para poder desarrollar posteriormente esta actividad. Una vez explicado el tema, para saber si los alumos han comprendido la materia impartida se evalúa el grado de adquisión de conocimiento básico antes de la experiencia TBL utilizando la aplicación Socrative donde se les pregunta cuestiones importantes relacionadas con la fisiología del sistema nervioso. Posteriormente se pasa al desarrollo de la actividad propuesta con la novedad de que en la preparación del seminario no se forman equipos independientes si no cooperativos.

La elaboración del seminario tiene un peso del 5\% de la nota final de la asignatura y se ha divido a su vez en tres fases que se describirán con detalle en los siguientes apartados:

\section{$1^{a}$ Fase (Organización de equipos inteligentes, reparto de tareas y búsqueda de información)}

Durante esta fase el profesor divide la clase en dos grupos, de modo que en cada una se van a preparar dos seminarios sobre dolor orofacial. A su vez, cada grupo (formado más o menos por la mitad de la clase) se divide en 4 equipos compuesto cada uno de ellos por 4 ó 5 alumnos, de modo que cada equipo se va a encargar de buscar la información referente a un apartado en los que se ha dividido el trabajo (en cada equipo se elige 1 coordinador, 1 portavoz y $2 / 3$ vocales).

El tema propuesto se divide en cuatro apartados: 1. Definición y tipos de dolor orofacial (según el estímulo, según la transmisión...), 2. Receptores y neuroquímica de la nocicepción

(cc) BY-NC-ND 2018, Universitat Politècnica de València

Congreso IN-RED (2018) 
orofacial (tipo, localización, estructura...), 3. Vías de transmisión y centro nervioso, 4. Tipos de tratamientos; el profesor asigna a cada equipo la elaboración de uno de ellos. Los apartados se han creado en función de conceptos estudiados previamente en clase sobre fisiología del sistema nervioso, concretamente sobre la fisiología del dolor.

En esta fase, el objetivo es que los alumnos de cada equipo sean capaces de buscar información y aplicar conocimientos previos sobre la somatocepción del dolor en general al caso específico de dolor orofacial y en concreto al apartado específico que les haya tocado. Por ejemplo, uno de los apartados es que definan dolor orofacial y enumeren que tipos de dolor hay según su origen. Para el desarrollo de este apartado cuentan de base con la definición de dolor y tipos que hay siendo lo que tienen que definir el caso específico de dolor orofacial. Para llevar a cabo este objetivo cada equipo de trabajo cuenta con un plazo de una semana fuera del aula. Se les da libertad para que usen los recursos que ellos crean conveniente como apuntes de clase, biblioteca, internet ....... y sentido común.

Durante el desarrollo del trabajo, cada equipo debe preparar un portfolio que contenga:

a) el plan de trabajo del equipo detallado

b) un documento que explique cuales han sido sus fuentes de información

c) un trabajo escrito con la información del apartado que le ha tocado desarrollar.

La entrega portfolio al profesor deberá realizarse por el coordinador de cada equipo.

\section{$2^{a}$ Fase (Elaboración del seminario en equipos cooperativos)}

Esta fase se desarrolla dentro del aula y los 8 equipos formados en cada clase ya tienen que tener preparada la información que les ha tocado. Dado que el trabajo está organizado en 4 apartados, en esta etapa los dos equipos encargados de buscar la misma información se deben juntar con el fin de poner en común la infomación recabada por separado. Así, la dinámica de esta fase consiste en crear grupos de expertos formados por los portavoces de ambos equipos. Estos alumnos se intercambian la información recogida y vuelven a sus grupos nodrizas para explicar a sus compañeros las nuevas aportaciones. Para finalizar, cuentan con 15 minutos para elaborar 2 diapositivas del apartado que les ha tocado.

\section{$3^{a}$ Fase (Presentación del seminario y evaluación del conocimiento adquirido)}

Una vez finalizada la fase de elaboración del seminario, cada grupo debe preparar una presentación con cada uno de los apartados que van a formar el seminario del dolor orofacial. De esta manera, utilizando la técnica de aprendizaje puzzle contaremos en cada clase con dos seminarios.

La presentación oral de cada seminario se realiza por uno de los vocales de cada equipo que haya participado en la elaboración del trabajo y tendrá lugar en el aula de cada clase de teoría con los medios disponibles, con una duración máxima de 10 minutos para cada grupo. Tras la presentación se abre un turno de preguntas y comentarios por parte del resto de equipos hacia el grupo que ha realizado la presentación (unos $5 \mathrm{~min}$ ). 
Una vez concluidas las presentaciones de los seminarios cada equipo valora qué seminario ha sido mejor ejecutado y por tanto le ha aportado más información. El resultado de dicha valoración por parte del alumnado se tiene en cuenta en la evaluación final de cada equipo.

Por último, para evaluar el grado de adquisición de nuevos conocimientos de manera individual generados tras las distintas competencias empleadas en la elaboración del seminario, se realiza una prueba de autoevaluación utilizando la aplicación Socrative donde se les pregunta cuestiones importantes relacionadas con los apartados que los estudiantes previamente han trabajado de manera colaborativa. El tiempo de la prueba no será de más de 30 min y el número de preguntas será de 2 a 3 por apartado.

\section{Resultados}

\section{Resultados de aprendizaje. Conocimiento básico antes de la experiencia TBL junto con el uso de técnicas de aprendizaje cooperativo.}

Antes de la elaboración del seminario por los equipos cooperativos se realizó una prueba de autoevaluación en la que participaron 60 de los 82 alumnos matriculados en el curso 2017/2018. Lo que corresponde aproximadamente con un 73,2\% de alumnos matriculados. La prueba consistía en 20 preguntas tipo test sobre fisiología general del sistema nervioso (temario que se impartió en clase previamente). Para la realización de esta prueba se utilizó la aplicación Socrative que nos facilitó un registro del porcentaje de preguntas acertadas por los alumnos de cada grupo de teoría. En la figura 1 se puede observar que la mayoría de los alumnos tienen entre un $80 \%$ y $85 \%$ de preguntas acertadas en ambos grupos, este resultado nos animó a poner en marcha la actividad propuesta.

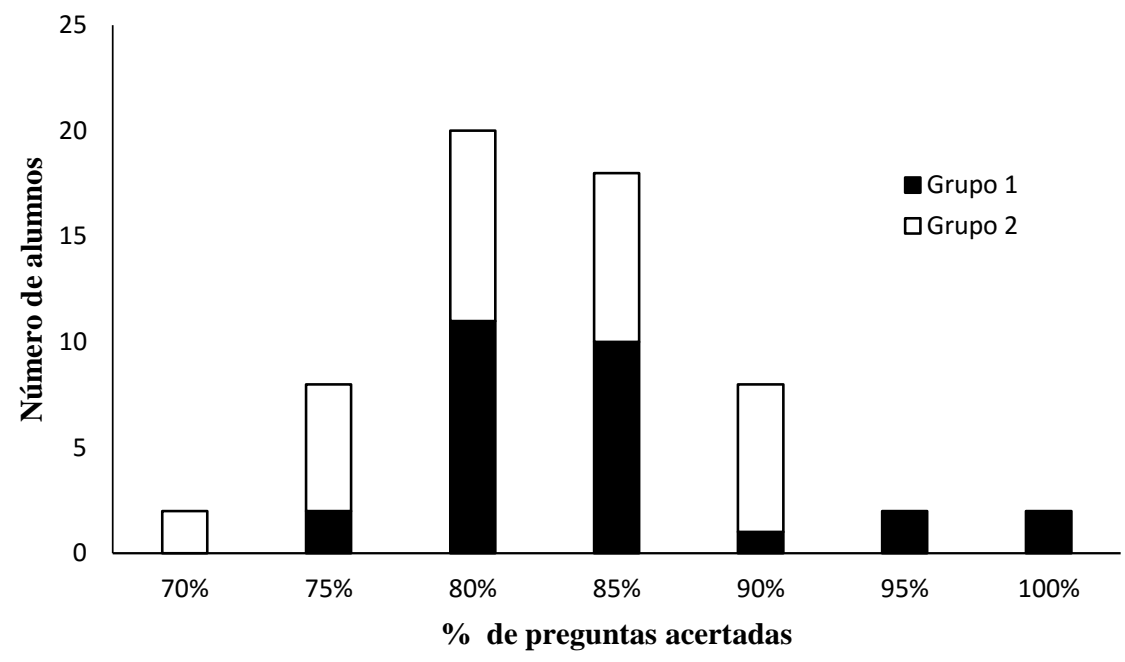

Figura 1. Porcentaje de preguntas acertadas sobre fisiología general por número de alumnos de cada grupo de teoría. Las columnas negras representan a los alumnos del grupo 1 y las blancas a los del grupo 2 . 
2. Resultados de aprendizaje. Conocimiento aplicado después de la experiencia TBL junto con el uso de técnicas de aprendizaje cooperativo.

De los 82 alumnos matriculados en los dos grupos del curso participaron en la actividad propuesta 62 alumnos (un 79,2\%). De esos 62 completaron las tres fases de la actividad 52 alumnos $(84 \%)$. Para ver si era eficaz este nuevo método de aprendizaje se realizaron dos evaluaciones diferentes: una durante el procedimiento, después del seminario realizado en la fase 3 del desarrollo de la actividad y una evaluación final en la prueba de evaluación continua donde a parte de las preguntas sobre fisiología general del sistema nervioso se incluyó también preguntas aplicadas relacionada con la actividad propuesta.

Los resultados de estas evaluaciones se muestran en los siguientes subapartados:

\subsection{Resultados de aprendizaje. Conocimiento aplicado durante la actividad.}

Para evaluar el conocimiento adquirido tras la presentación del seminario se realizó una primera prueba de evaluación donde se plantearon dos preguntas cortas, dos preguntas de verdadero/falso y dos preguntas de elección múltiple. Para el registro de las repuestas por parte de los alumnos se utilizó la aplicación Socrative. Los resultados se muestran en la figura 2, donde se puede observar que la mayor parte de los alumnos de ambos grupos contestaron bien el $100 \%$ de las preguntas planteadas.

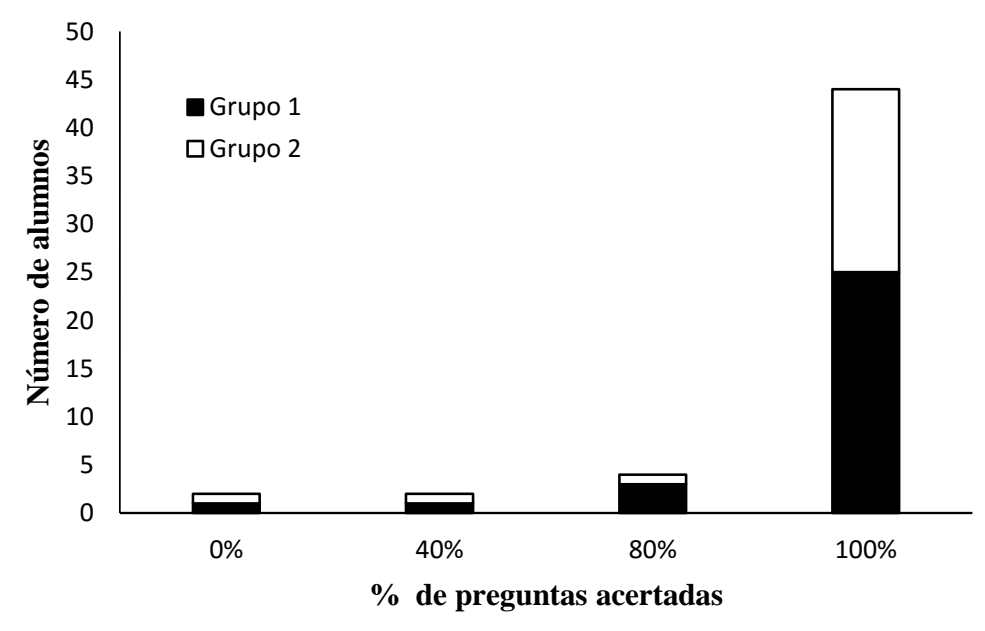

Figura 2. Porcentaje de preguntas acertadas sobre el dolor orofacial por número de alumnos de cada grupo de teoría. Las columnas negras representan a los alumnos del grupo 1 y las blancas a los del grupo 2. 
Generación colaborativa de conocimiento. Una experiencia de aprendizaje basada en equipos o Team Based Learning (TBL) en la práctica odontológica.

\subsection{Resultados de aprendizaje. Conocimiento aplicado en la prueba de evaluación continua}

Para la evaluación formativa, que se realiza después del aprendizaje se utilizó la prueba de evaluación continua dónde además de las preguntas sobre el temario de la asignatura se incluyó una pregunta sobre la actividad desarrollada (fue una pregunta tipo test con cuatro posibles respuestas). Dado que cada curso consta de dos grupo de teoría lo primero que quisimos ver es si ambos grupos se comportaban de manera homogénea, para ello analizamos el resultado del aprendizaje por separado como describimos a continuación:

A la prueba de evaluación continua se presentaron 36 alumnos de los 40 matriculados en grupo 1 y 33 alumnos de los 42 matriculados en el grupo 2. En total el $84 \%$ de los alumnos matriculados en curso actual. Los resultados en el grupo 1 fueron los siguientes: un 83,3\% de los alumnos contestaron bien, un 13,9\% contestaron mal y un 2,8 \% dejaron la pregunta sin contestar. En el caso del grupo 2 un 78,8 \% de los alumnos contestaron bien, un 12,1\% mal y un 9,10\% dejaron la pregunta sin contestar. Cuando se compararon los resultados de ambos grupos no existían diferencias significativas entre ellos como era de esperar ya que en ambos grupos se utilizó la misma metodología de aprendizaje. Estos resultados se muestran la figura 3.

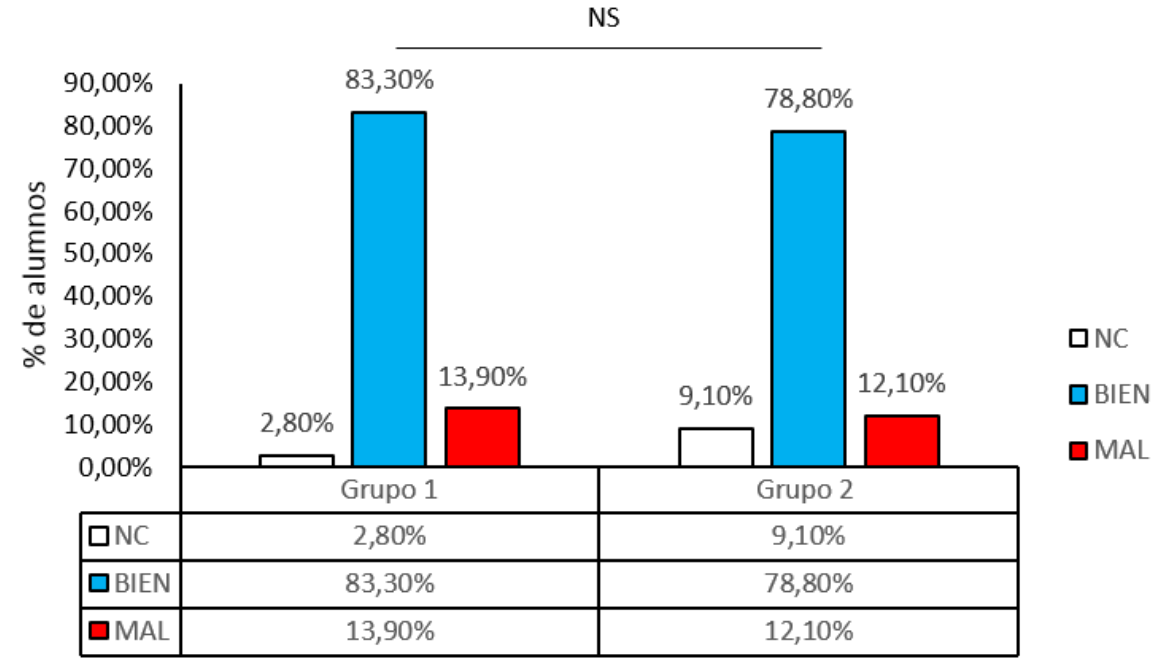

Figura 3. Porcentaje alumnos que no contestan (NC), que contestan bien (BIEN) y que contestan mal (MAL) a la pregunta sobre el dolor orofacial en la prueba de evaluación en el grupo 1 y el grupo 2 del curso 2017/2018. Las barras representan el porcentaje de alumnos que NC, que contestan bien o que contestan mal a la pregunta del dolor orofacial en relación al número total de individuos analizados en cada grupo. NS $=$ no se encontraron diferencias significativas cuando se realizó la prueba $\chi^{2}$ de Pearson.

Una vez demostrada la homogenidad entre los grupos se agruparon ambos en uno solo llamado curso (2017/18).

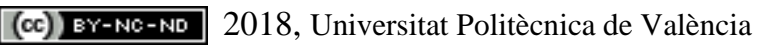

Congreso IN-RED (2018) 


\section{Comparación de los resultados de aprendizaje de conocimiento aplicado utilizado la metodología nueva y la tradicional.}

Por último, para probar que la nueva metodología de aprendizaje propuesta en esta memoria es realmente útil comparamos los resultados de aprendizaje de conocimiento aplicado obtenidos en la prueba de evaluación continua del curso actual (2017/2018) con los resultados obtenidos en la prueba de evaluación continua de los alumnos del curso anterior (2016/2017).

La diferencia entre ambos cursos fue que en el curso 2016/2017 la elaboración del seminario de dolor orofacial se hizo de forma tradicional, es decir, sin aplicar las nuevas técnicas de aprendizaje cooperativo (equipos inteligentes, grupo de expertos...) mientras que en el curso actual 2017/2018 se utilizó la metodología descrita en esta memoria.

$\mathrm{Al}$ analizar los resultados de la prueba de evaluación continua de ambos cursos donde se les realizo una pregunta parecida sobre el dolor orofacial pudimos observar que en el curso anterior un $20 \%$ de los alumnos contestaron bien, un $54 \%$ contestaron mal y un $26 \%$ dejaron la pregunta sin contestar. En el caso del curso actual un $81,2 \%$ de los alumnos contestaron bien, un $13 \%$ mal y un 5,8 \% dejaron la pregunta sin contestar (figura 4). La prueba $\chi^{2}$ de Pearson demostró que hay diferencias significativas entre el curso 2016/2017 y el curso 2017/2018 ( $<<0,0001$ ). Este resultado prueba que la metodología actual es más eficaz que la tradicional aumentando el aprendizaje de conocimiento aplicado por parte del alumnado.

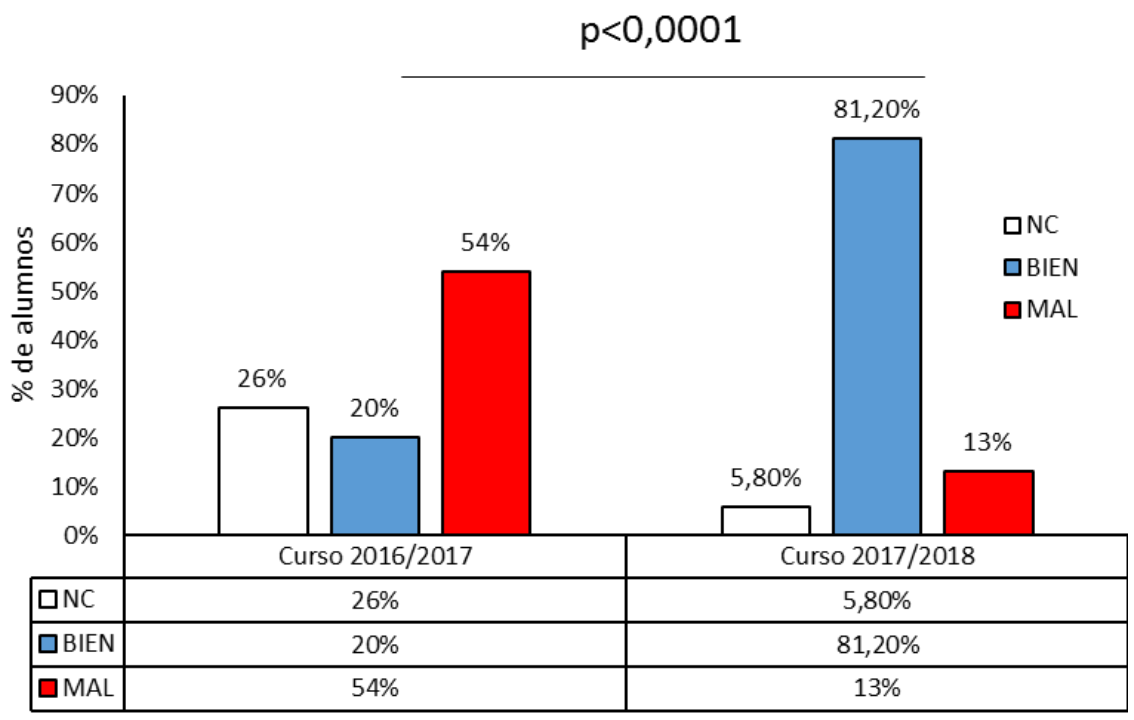

Figura 4. Porcentaje alumnos que no contestan (NC), que contestan bien (BIEN) y que contestan mal (MAL) a la pregunta sobre el dolor orofacial en la prueba de evaluación del curso 2016/2017 y del curso 2017/2018. Las barras representan el porcentaje de alumnos que NC, que contestan bien o que contestan mal a la pregunta del dolor orofacial en relación al número total de individuos analizados en cada curso académico.

\section{Grado de satisfacción de los alumnos con la nueva metodología.}


Generación colaborativa de conocimiento. Una experiencia de aprendizaje basada en equipos o Team Based Learning (TBL) en la práctica odontológica.

Para valorar la acogida de los alumnos a la nueva metodología de aprendizaje se les paso una encuesta con cuatro preguntas para ver la opinión de los alumnos sobre tiempo empleado, trabajo en equipo, el aprendizaje de nuevos conocimientos y si les había gustado más o menos que otras metodologías. En la encuesta participaron el $79 \%$ de los alumnos que completaron las tres fases de la actividad. Los resultados de las preguntas de la encuesta se muestran a continuación.

En la Figura 5 se recoge los resultados de la primera pregunta de la encuesta en la que se evaluó el esfuerzo percibido por los alumnos respecto al desarrollo de esta actividad. Con este fin se les preguntó si fué mucho el tiempo que invirtieron fuera del aula para su desarrollo. La mayor parte de los alumnos, el $66 \%$, contestaron que no les llevo mucho tiempo, un $27 \%$ contestaron que el tiempo de desarrollo fue intermedio y solo un pequeño porcentaje de alumnos consideraron que les supuso mucho tiempo.

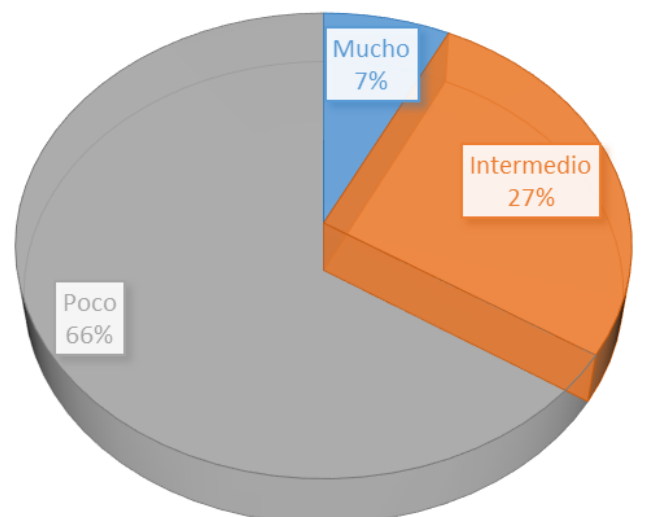

Figura 5. Tiempo requerido por el alumno para realizar la actividad.

Para saber si el trabajo en grupo fue una experiencia positiva para el alumno se les pregunto si les había gustado trabajar en equipo. La mayoría de los alumnos contestaron de manera afirmativa; incluso un porcentaje notable destaco que les había gustado mucho. Solo un porcentaje bajo contestó que preferían trabajar de manera individual, como podemos observar en la figura 6.

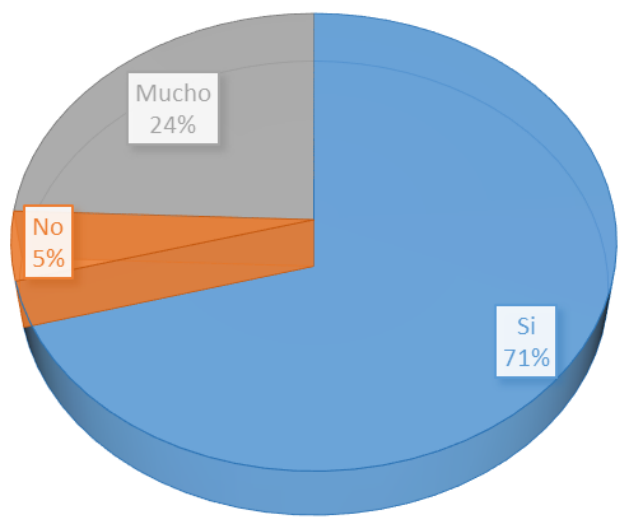

Figura 6. Grado de satisfacción del alumno por el trabajo en equipo.

(cc) EY-NC-ND 2018, Universitat Politècnica de València

Congreso IN-RED (2018) 
Respecto a la pregunta en la que se les consulta si consideraban positiva esta actividad en cuanto a su aprendizaje, como se puede ver en la Figura 8, la mayor parte de los alumnos contestaron de manera afirmativa.

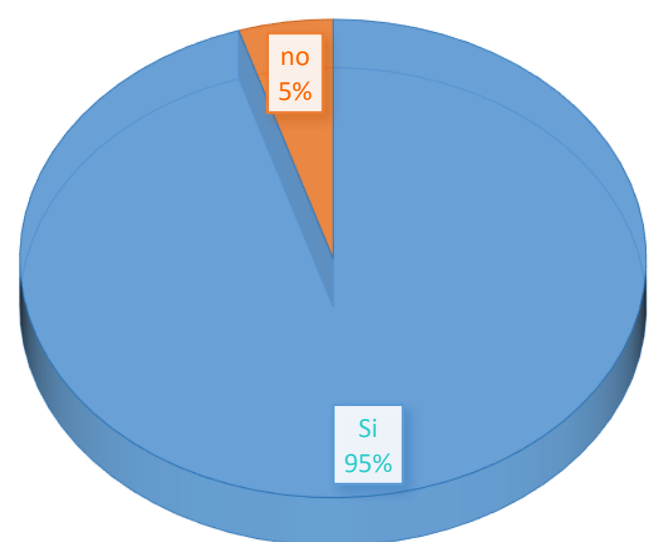

Figura 8. Aprendizaje de nuevo conocimiento.

Para finalizar, quisimos conocer la preferencia de los alumnos sobre el uso de la nueva metodología de aprendizaje versus a la antigua. La mayor parte de los alumnos prefieren la nueva metodología y sólo una pequeña parte de los alumnos consideraron que les gustaba más la antigua o las dos por igual (figura 9). Entre los comentarios de los alumnos queremos destacar la opinión de que el trabajo en equipos cooperativos es un buen método para aprender ya que fomenta el compañerismo, reduce el tiempo de trabajo y les ayuda a resolver dudas.

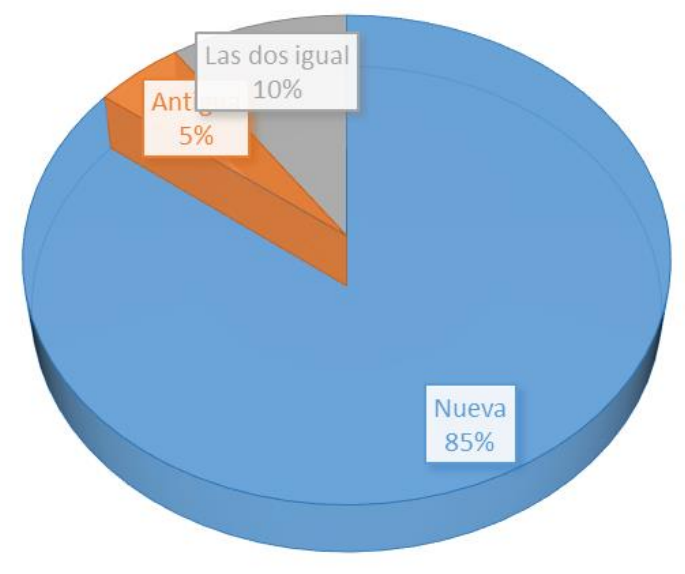

Figura 9. preferencia de los alumnos sobre el uso de la nueva metodología de aprendizaje versus a la antigua 


\section{Conclusión}

Hemos diseñado y realizado una nueva actividad formativa para que los alumnos participen más a la hora de generar nuevos conocimientos a partir de los conocimientos previos y pierdan el miedo a resolver preguntas aplicadas. El análisis de los resultados obtenidos indica que esta nueva metodología incrementa de manera significativa el porcentaje de acierto en preguntas aplicadas en pruebas de evaluación continua con respecto a otros cursos donde no se había puesto en marcha esta actividad, poniendo de manifiesto la eficacia del método. Además, podemos concluir que la nueva metodología de aprendizaje minimiza el tiempo de trabajo fuera del aula, aumenta la participación activa del alumno en la adquisición de nuevos conocimientos, fomenta el compañerismo y los alumnos la eligen frente a otras metodologías. La eficacia del método en el aprendizaje junto a la aceptación del mismo por el alumnado nos anima a seguir trabajado en esta dirección en los futuros cursos académicos.

\section{Referencias}

ARCEO FDB, ROJAS GH, GONZÁLEZ ELG (2002). Estrategias docentes para un aprendizaje significativo: una interpretación constructivista. Libro electrónico: mapas.eafit.edu.co.

BARR, R.B Y TAGG, J. (1995). "From teaching to learning. A new paradigm for undergraduate education", en Change, 27 (6), pp. 13-25. Revista electrónica de Tecnología Educativa, $n^{\circ} 29$

ESCUDERO E, SÁNCHEZ-VERA I, BARHOUM R, PUCHE J E, MUÑOZ U (2017). Análisis del uso de autoevaluaciones en una plataforma digital en el entorno de la Fisiología Médica. Doi: http://dx.doi.org/10.4995/INRED2017.2017.6809

GARCÍA VALCÁRCEL, A. (1993). “Análisis de los modelos de enseñanza empleados en el ámbito universitario”, en Revista Española de Pedagogía, 194, 27-53

GARGALLO, B, GARCÍA- FÉLIX , I, MORERA, C. Y BENAVENT, A. (2015). Métodos innovadores y enfoques de aprendizaje en estudiantes universitarios. Doi:http://dx.doi.org/10.4995/INRED2015.2015.1576

JENSEN E, VILLALBA A (2003) Cerebro y aprendizaje: competencias e implicaciones educativas. Sumario Editorial

LARRY MICHAELSEN, ARLETA BAUMAN AND L. DEE FINK (2004). Team-Based Learning: A Transformative Use of Small Groups in College Teaching. Stylus Publishing (VA)

MACHEMER, P. L. . Y CRAWFORD, P. (2007). "Student perceptions of active learning in a large crossdisciplinary classroom", en Active Learning in Higher Education, 8 (1), 930.

MICHAELSEN, L.K.; SWEET, M. (2009) "The essential elements of team-based learning", Team-Based Learning: Small Group Learning's Next Big Step. New Directions in Teaching and Learning, p. 7-27. 
MOLERO LÓPEZ-BARJAS D., RUIZ CARRASCOSA J (2005). La evaluación de la docencia universitaria. Dimensiones y variables más relevantes. Revista de Investigación Educativa 23 (1)

MONEREO C. Y POZO J. I. (2003). La universidad ante la nueva cultura educativa. Enseñar y aprender para la autonomía. Madrid: Síntesis

RICARD, MATTHIEU (2010). "Educación: Promueve la cooperación, no la competitividad". http://www.matthieuricard.org/es/blog/posts/educacion-promueve-lacooperacion-no-la-competitividad [Consulta: 14 de marzo de 2017].

SÁNCHEZ-VERA I, ESCUDERO E, MUÑOZ U, BORREGO, M J, BARHOUM R (2017). Experiencia en la elaboración de videos didácticos por alumnos de Fisiología como parte de su proceso de aprendizaje. Doi: http://dx.doi.org/10.4995/INRED2017.2017.6819

SOFÍA ESTELLES-MIGUELA, JOSÉ MIGUEL ALBARRACÍN GUILLEMB, MARTA ELENA PALMER GATO Y MARTA PERIS-ORTIZ (2017) "Desarrollo de la Competencia de Trabajo en Equipo en la Asignatura de Dirección de Producción y Operaciones". Doi: http://dx.doi.org/10.4995/INRED2017.2017.6892

ZABALZA BERAZA, M.A. (2012). El estudio de las "buenas prácticas" docentes en la enseñanza universitaria. Revista de Docencia Universitaria. REDU, 10 (1), 17-42. Extraído el 2 de abril, 2012 de http://redaberta.usc.es/redu/index.php/REDU/article/view/363. 